\title{
LEVEL SETS OF MULTIPLE ERGODIC AVERAGES
}

\author{
AI-HUA FAN, LINGMIN LIAO, AND JI-HUA MA
}

\begin{abstract}
We propose to study multiple ergodic averages from multifractal analysis point of view. In some special cases in the symbolic dynamics, Hausdorff dimensions of the level sets of multiple ergodic average limit are determined by using Riesz products.
\end{abstract}

\section{INTRODUCTION}

Let $(X, T)$ be a topological dynamical system and let $\ell \geq 2$ be a positive integer. We consider the following multiple ergodic averages

$$
\frac{1}{n} \sum_{k=1}^{n} f_{1}\left(T^{k} x\right) f_{2}\left(T^{2 k} x\right) \cdots f_{\ell}\left(T^{\ell k} x\right),
$$

where $f_{1}, \cdots, f_{\ell}$ are $\ell$ given continuous functions. Such multiple ergodic averages were introduced and studied by Furstenberg [9] in his ergodic theoretic proof of Szemerédi's theorem on arithmetic progressions. Since then these averages have received extensive studies in various contexts. For example, the $L^{2}$-normal convergence of (1.1) is proved by Host and Kra [11] with respect to a given invariant measure, and the almost sure convergence is proved earlier by Bourgain [2] in the case of $\ell=2$. In this note we propose to study these multiple ergodic averages from multifractal analysis point of view.

Multifractal analysis of ergodic averages concerns the Hausdorff dimension of the level sets of the ergodic average limit. It reflects the complex behavior of the underlying chaotic dynamical system. There was a wide study in the case of simple ergodic averages $(\ell=1)$ in the last decades $([6,7,8,13,14,15,16])$. Our first investigation shows that the multifractal analysis of multiple ergodic averages $(\ell \geq 2)$ is much more difficult. This note aims at a special case where $X$ is the symbolic space $\mathbb{D}=\{+1,-1\}^{\mathbb{N}}$ (N denoting the set of positive integers) and the dynamics is defined by the shift transformation $T:\left(x_{1}, x_{2}, \cdots\right) \mapsto\left(x_{2}, x_{3} \cdots\right)$. The metric on $\mathbb{D}$ is chosen to be

$$
\rho(x, y)=2^{-\min \left\{k \geq 1: x_{k} \neq y_{k}\right\}} \text { for } x, y \in \mathbb{D} .
$$

The Hausdorff dimension of a set $A$ will be denoted by $\operatorname{dim}_{H} A$. See 3 for notions of dimensions of a set and [4 for notions of dimensions of a measure. Let $\ell \geq 1$. We shall examine the averages (1.1) with the functions

$$
f_{1}(x)=f_{2}(x)=\cdots=f_{\ell}(x)=x_{1} \text { for } x \in \mathbb{D} .
$$

2010 Mathematics Subject Classification. Primary 37C45, 42A55; Secondary 37A25, 37D35.

Key words and phrases. Multiple ergodic averages, Hausdorff dimension, Riesz product. 
Then for $\theta \in[-1,1]$, we consider the level set

$$
B_{\theta}:=\left\{x \in \mathbb{D}: \lim _{n \rightarrow \infty} \frac{1}{n} \sum_{k=1}^{n} x_{k} x_{2 k} \cdots x_{\ell k}=\theta\right\} .
$$

We prove the following result.

Theorem 1.1. For any $\theta \in[-1,1]$, we have

$$
\operatorname{dim}_{H}\left(B_{\theta}\right)=1-\frac{1}{\ell}+\frac{1}{\ell} H\left(\frac{1+\theta}{2}\right),
$$

where $H(t)=-t \log _{2} t-(1-t) \log _{2}(1-t)$ is the entropy function.

This result was known to Besicovitch and Eggleston when $\ell=1$. Remark that the Hausdorff dimension of $B_{\theta}$ is strictly positive for any $\theta \in[0,1]$ when $\ell \geq 2$. Actually,

$$
\operatorname{dim}_{H} B_{\theta} \geq 1-1 / \ell>0 \quad \text { if } \quad \ell \geq 2 .
$$

The proof of the theorem is based on the fact that $\mathbb{D}$ has a group structure and the functions $x \mapsto x_{k} x_{2 k} \cdots x_{\ell k}$ are group characters and even they constitute a dissociated set of characters in the sense of Hewitt-Zuckermann [10. As we shall show, the set $B_{\theta}$ supports a Riesz product, a nice measure which has the same Hausdorff dimension as that of $B_{\theta}$. The idea of using Riesz product is inspired by [5] where oriented walks were studied. Although the Riesz product works perfectly for the above case concerned by Theorem 1.1 it has its limit for the general case.

We point out that the situation seems very different when the functions in (1.2) are replaced by other functions. For example, when $f_{i}$ are chosen as $\left(x_{1}+1\right) / 2$ which takes 0 and 1 as values. The obtained set can be identified with

$$
A_{\theta}:=\left\{x \in\{0,1\}^{\mathbb{N}}: \lim _{n \rightarrow \infty} \frac{1}{n} \sum_{k=1}^{n} x_{k} x_{2 k} \cdots x_{\ell k}=\theta\right\} .
$$

The set $A_{\theta}$ is similar to $B_{\theta}$, but the determination of its dimension is more difficult.

Actually, we are motivated by the study of $A_{\theta}$. The Riesz product method is not adapted to it. Then we propose to looking at the following set

$$
X_{0}:=\left\{x \in\{0,1\}^{\mathbb{N}}: x_{n} x_{2 n}=0, \quad \text { for all } n\right\},
$$

which is a subset of $A_{\theta}$ with $\ell=2$ and $\theta=0$. We obtain the box dimension (denoted by $\operatorname{dim}_{B}$ ) for $X_{0}$ by a combinatoric method.

Theorem 1.2. Let $\left\{a_{n}\right\}$ be the Fibonacci sequence defined by

$$
a_{0}=1, a_{1}=2, \quad a_{n}=a_{n-1}+a_{n-2}(n \geq 2) .
$$

We have

$$
\operatorname{dim}_{B}\left(X_{0}\right)=\frac{1}{2 \log 2} \sum_{n=1}^{\infty} \frac{\log a_{n}}{2^{n}}=0.8242936 \cdots .
$$

The problem of determining the Hausdorff dimension of $X_{0}$ is now solved by Kenyon, Peres and Solomyak [12, where a class of sets similar to $X_{0}$ is studied. The result in 12 together with Theorem 1.2 shows that $\operatorname{dim}_{H} X_{0}<\operatorname{dim}_{B} X_{0}$. 


\section{RIESZ PRODUCTS}

Let us consider $\mathbb{D}$ as an infinite product group of the multiplicative group $\{+1,-1\}$. The dual group of $\mathbb{D}$ consists of the Walsh functions $\left\{w_{n}(x)\right\}_{n=0}^{\infty}$ defined as follows. Define $w_{0}=1$. For each $n \geq 1$, let

$$
n=2^{n_{1}-1}+2^{n_{2}-1}+\cdots+2^{n_{s}-1}, \quad 1 \leq n_{1}<n_{2}<\cdots<n_{s},
$$

be the unique expansion of the integer $n$ in base 2 . Then we define

$$
w_{n}(x)=x_{n_{1}} x_{n_{2}} \cdots x_{n_{s}} .
$$

An important subset of Walsh functions is the set of the Rademacher functions $\left\{r_{n}(x)\right\}_{n=1}^{\infty}$ defined by $r_{n}(x)=x_{n}$. The Rademacher functions are mutually independent with expectation zero with respect to the Haar measure. The following immediate consequence of the independence will be frequently used in the sequel.

Lemma 2.1. Let $f$ and $g$ be two Haar integrable functions on $\mathbb{D}$. Suppose that $f$ depends only on the first $n$ coordinates of $x$ and $g$ is independent of the first $n$ coordinates. Then

where $d x$ stands for the Haar measure on $\mathbb{D}$.

$$
\int f(x) g(x) d x=\int f(x) d x \int g(x) d x
$$

The $n$-th Fourier coefficient of an integrable function $f$ is defined by

$$
\hat{f}(n)=\int f(x) w_{n}(x) d x .
$$

In the follows, we shall denote

$$
\xi_{k}(x)=x_{k} x_{2 k} \cdots x_{\ell k} \quad \text { for all } k \geq 1 .
$$

Consider the product

$$
d P_{\theta}(x)=\prod_{k=1}^{\infty}\left(1+\theta \xi_{k}(x)\right) d x .
$$

The following lemma shows that the above product defines a probability measure on $\mathbb{D}$, which will be called Riesz product.

Lemma 2.2. The partial products of the above infinite product converge in the weak-* topology to a probability measure $P_{\theta}$. Furthermore, for any function $f$ depending only on the first $n$ coordinates of $x$, we have

$$
\mathbb{E}_{\theta}[f]=\int f(x) \prod_{k=1}^{\lfloor n / \ell\rfloor}\left(1+\theta \xi_{k}(x)\right) d x,
$$

where $\mathbb{E}_{\theta}[\cdot]$ stands for the expectation with respect to $P_{\theta}$ and " $\lfloor\cdot\rfloor$ " is the integer part function.

Proof For $N \geq 1$, let

$$
P_{N}(x)=\prod_{k=1}^{N}\left(1+\theta \xi_{k}(x)\right) .
$$

Then

$$
P_{N+1}(x)-P_{N}(x)=\theta P_{N}(x) \xi_{N+1}(x) .
$$


Observe that for the fixed Walsh function $w_{n}(x)=x_{n_{1}} x_{n_{2}} \cdots x_{n_{s}}$, by Lemma 2.1 one has

$$
\int P_{N}(x) \xi_{N+1}(x) w_{n}(x) d x=0
$$

whenever $(N+1) \ell>n_{s}$. It follows that $\hat{P}_{N}(n)=\hat{P}_{N+1}(n)$ for large $N$, so the limit

$$
\lim _{N \rightarrow \infty} \int P_{N}(x) w_{n} d x
$$

exists. That is to say, the measures $P_{N}(x) d x$ converge weakly to a limit measure $P_{\theta}$.

The formula (2.1) follows directly from Lemma 2.1 and the definition of the Riesz product $P_{\theta}$ as a weak limit.

The functions $\xi_{n}$ are not $P_{\theta}$-independent, but they are orthogonal. Therefore, we can get the following law of large numbers.

Lemma 2.3. Suppose that $g$ is a function on the interval $[-1,1]$ such that

$$
g(t)=\sum_{n=0}^{\infty} g_{n} t^{n} \quad \text { with } \quad \sum_{n=1}^{\infty}\left|g_{n}\right|<\infty .
$$

Then for $P_{\theta}$-almost all $x$,

$$
\lim _{n \rightarrow \infty} \frac{1}{n} \sum_{k=1}^{n} g\left(\xi_{k}(x)\right)=\mathbb{E}_{\theta}\left[g\left(\xi_{1}\right)\right]
$$

Proof Notice that $\xi_{k}^{2 n}(x)=1$ and $\xi_{k}^{2 n-1}(x)=\xi_{k}(x)$ for any integer $n \geq 1$. Then we get

$$
g\left(\xi_{k}\right)=\sum_{n=0}^{\infty} g_{2 n}+\xi_{k} \sum_{n=1}^{\infty} g_{2 n-1}
$$

By the formula (2.1), we have

$$
\mathbb{E}_{\theta}\left(\xi_{k}\right)=\theta, \quad \mathbb{E}_{\theta}\left(\xi_{j} \xi_{k}\right)=\theta^{2}, \quad(j \neq k)
$$

It follows that

$$
\mathbb{E}_{\theta}\left[g\left(\xi_{k}\right)\right]=\sum_{n=0}^{\infty} g_{2 n}+\theta \sum_{n=1}^{\infty} g_{2 n-1}, \quad \operatorname{Cov}_{\theta}\left[g\left(\xi_{j}\right), g\left(\xi_{k}\right)\right]=0 \quad(j \neq k) .
$$

Therefore, the system $g\left(\xi_{k}\right)-\mathbb{E}_{\theta}\left[g\left(\xi_{k}\right)\right](k=1,2, \cdots)$ is orthogonal in $L^{2}\left(P_{\theta}\right)$. By the Menchoff Theorem $([17])$, the series

$$
\sum_{k=0}^{\infty} \frac{1}{k}\left(g\left(\xi_{k}\right)-\mathbb{E}_{\theta}\left[g\left(\xi_{k}\right)\right]\right)
$$

converges $P_{\theta}$-almost surely. Now the desired result follows from Kronecker's theorem. 


\section{Proof of Theorem 1.1}

Applying Lemma 2.3 to $g(t)=t$, we get that for $P_{\theta}$-almost all $x$,

$$
\lim _{m \rightarrow \infty} \frac{1}{m} \sum_{k=1}^{m} \xi_{k}(x)=\mathbb{E}\left(\xi_{1}\right)=\theta .
$$

This means that the Riesz product $P_{\theta}$ is supported by the set $B_{\theta}$. Now we are going to compute the local dimension of the Riesz product $P_{\theta}$ and we will apply Billingsley's theorem to conclude Theorem 1.1 .

For each $x \in \mathbb{D}$ and $n \geq 1$, let

$$
I_{n}(x)=I\left(x_{1}, \cdots, x_{n}\right)=\left\{y \in \mathbb{D}: y_{k}=x_{k} \text { for } 1 \leq k \leq n\right\} .
$$

It is the $n$-cylinder containing $x$, a ball of diameter $2^{-n}$. By the formula (2.1), for any $n \geq \ell$, we have

$$
P_{\theta}\left(I_{n}(x)\right)=\frac{1}{2^{n}} \prod_{k=1}^{\lfloor n / \ell\rfloor}\left(1+\theta \xi_{k}(x)\right) .
$$

Recalling that $\xi_{k}(x)=+1$ or -1 for all $x$, by Taylor formula, we have

$$
\log \left(1+\theta \xi_{k}(x)\right)=-\sum_{n=1}^{\infty} \frac{\theta^{2 n}}{2 n}+\sum_{n=1}^{\infty} \frac{\theta^{2 n-1}}{2 n-1} \xi_{k}(x) .
$$

Then for all points $x \in B_{\theta}$,

$$
\lim _{m \rightarrow \infty} \frac{1}{m} \sum_{k=1}^{m} \log \left(1+\theta \xi_{k}(x)\right)=-\sum_{n=1}^{\infty} \frac{\theta^{2 n}}{2 n}+\sum_{n=1}^{\infty} \frac{\theta^{2 n-1}}{2 n-1} \theta .
$$

The right hand side can be written as

$$
\theta \log (1+\theta)-\frac{\theta-1}{2} \log \left(1-\theta^{2}\right)=\left[1-H\left(\frac{1+\theta}{2}\right)\right] \log 2 .
$$

It then follows that for all points $x \in B_{\theta}$,

$$
\lim _{n \rightarrow \infty} \frac{\log P_{\theta}\left(I_{n}(x)\right)}{\log \left|I_{n}(x)\right|}=\lim _{n \rightarrow \infty} \frac{\sum_{k=1}^{\lfloor n / \ell\rfloor} \log \left(1+\theta \xi_{k}(x)\right)-\log 2^{n}}{\log 2^{-n}}=1-\frac{1}{\ell}+\frac{1}{\ell} H\left(\frac{1+\theta}{2}\right) .
$$

The proof is completed by applying Billingsley's theorem ([1]).

\section{Proof of Theorem 1.2}

It is clear that

$$
\operatorname{dim}_{B} X_{0}=\lim _{n \rightarrow \infty} \frac{\log _{2} N_{n}}{n}
$$

if the limit exists, where $N_{n}$ is the cardinality of the following set

$$
\left\{\left(x_{1} x_{2} \cdots x_{n}\right): x_{\ell} x_{2 \ell}=0 \text { for } \ell \geq 1 \text { such that } 2 \ell \leq n\right\} .
$$

Each equality $x_{\ell} x_{2 \ell}=0$ defines a condition on the sequence $\left(x_{1} \cdots x_{n}\right)$ which determines the cylinder $I\left(x_{1}, \cdots, x_{n}\right)$. We observe that all these conditions can be 
divided into "independent" groups of conditions. Let

$$
\begin{aligned}
C_{0} & :=\left\{1,3,5, \ldots, 2 n_{0}-1\right\}, \\
C_{1} & :=\left\{2 \cdot 1,2 \cdot 3,2 \cdot 5, \ldots, 2 \cdot\left(2 n_{1}-1\right)\right\}, \\
& \cdots \\
C_{k} & :=\left\{2^{k} \cdot 1,2^{k} \cdot 3,2^{k} \cdot 5, \ldots, 2^{k} \cdot\left(2 n_{k}-1\right)\right\}, \\
& \cdots \\
C_{m} & :=\left\{2^{m} \cdot 1\right\},
\end{aligned}
$$

where $n_{k}$ is the biggest integer such that

$$
2^{k}\left(2 n_{k}-1\right) \leq n, \quad \text { i.e., } \quad n_{k}=\left\lfloor\frac{n}{2^{k+1}}+\frac{1}{2}\right\rfloor
$$

and $m$ is the biggest integer such that

$$
2^{m} \leq n, \quad \text { i.e., } \quad m=\left\lfloor\log _{2} n\right\rfloor .
$$

We have the decomposition $\{1, \cdots, n\}=C_{0} \sqcup C_{1} \sqcup \cdots \sqcup C_{m}$ and

$$
n_{0}>n_{1}>\cdots>n_{m-1}>n_{m}=1 \text {. }
$$

The conditions $x_{\ell} x_{2 \ell}=0$ with $\ell$ in different columns in the table defining $C_{0}, \cdots, C_{m}$ are independent. We are going to use this independence to count the number of possible choices for $\left(x_{1}, \cdots, x_{n}\right)$.

We have $n_{m}(=1)$ columns each of which has $m+1$ elements. Then we have $a_{m+1}$ choices for $x_{\ell}$ with $\ell$ in the first column since $\left(x_{\ell}, x_{2 \ell}\right)$ is conditioned to be different from $(1,1)$. Each of the next $n_{m-1}-n_{m}$ columns has $m$ elements, then we have $a_{m}^{n_{m-1}-n_{m}}$ choices for the $x_{\ell}$ 's with $\ell$ in these columns. By induction, we get

$$
N_{n}=a_{m+1}^{n_{m}} a_{m}^{n_{m-1}-n_{m}} a_{m-1}^{n_{m-2}-n_{m-1}} \cdots a_{1}^{n_{0}-n_{1}} .
$$

Now, the box dimension of the set $X_{0}$ equals to

$$
\begin{aligned}
\lim _{n \rightarrow \infty} \frac{\log _{2} N_{n}}{n} & =\lim _{n \rightarrow \infty} \frac{1}{n}\left(n_{m} \log _{2} a_{m+1}+\sum_{k=0}^{m}\left(n_{k-1}-n_{k}\right) \log _{2} a_{k}\right) \\
& =\lim _{n \rightarrow \infty} \frac{1}{n}\left(\log _{2} a_{m+1}+\sum_{k=0}^{\left\lfloor\log _{2} n\right\rfloor}\left(\left\lfloor\frac{n}{2^{k}}+\frac{1}{2}\right\rfloor-\left\lfloor\frac{n}{2^{k+1}}+\frac{1}{2}\right\rfloor\right) \log _{2} a_{k}\right) \\
& =\sum_{k=1}^{\infty} \frac{\log _{2} a_{k}}{2^{k+1}} .
\end{aligned}
$$

Acknowledgement. This work is partially supported by NSFC10771164 (Ji-Hua Ma) and NSFC10901124 (Lingmin Liao).

\section{REFERENCES}

[1] P. Billingsley, Ergodic theory and information, John Wiley and Sons, Inc., New York-LondonSydney, (1965).

[2] J. Bourgain, Double recurrence and almost sure convergence. J. Reine. Angew. Math. 404 (1990), 140-161.

[3] K.J. Falconer, Fractal Geometry : Mathematical Foundations and Applications, 2nd Edition. Wiley, 2003.

[4] A.H. Fan, Sur les dimension de measure, Studia Math., 111 (1994), 1-17. 
[5] A.H. Fan, Individual behaviors of oriented walks, Stoc. Proc. Appl., Stoc. Proc. Appl., 90 (2000) 263-275.

[6] A.H. Fan and D.J. Feng, On the distribution of long-term time averages on symbolic space, J. Stat. Phys., 99 (2000), no. 3-4, 813-856.

[7] A.H. Fan, D.J. Feng and J. Wu, Recurrence, dimension and entropy, J. London Math. Soc. (2), 64 (2001), no. 1, 229-244.

[8] A.H. Fan, L.M. Liao and J. Peyrière, Generic points in systems of specification and Banach valued Birkhoff ergodic average, Discrete Contin. Dyn. Syst., 21 (2008) 1103-1128.

[9] H. Furstenberg, Ergodic behavior of diagonal measures and a theorem of Szemerédi on arithmetic progressions. J. d'Analyse Math. 31 (1977), 204-256.

[10] E. Hewitt and H.S. Zuckerman, Singular measures with absolutely continuous convolution squares, Proc. Camb. Phil. Soc. 62 (1966), p. 399-420.

[11] B. Host and B. Kra, Nonconventional ergodci averages and nilmanifolds. Ann. Math. 161 (2005), 397-488.

[12] R. Kenyon, Y. Peres and B. Solomyak, Hausdorff dimension for fractals invariant under the multiplicative integers. preprint, 2011.

[13] J.H. Ma and Z.Y. Wen, Besicovitch subsets of self-similar sets, Ann. Inst. Fourier (Grenoble), 52 (2002), 1061-1074.

[14] E. Olivier, Multifractal analysis in symbolic dynamics and distribution of pointwise dimension for g-measures, Nonlinearity, 12 (1999), 1571-1585.

[15] Y. Pesin, Dimension theory in dynamical systems, University of Chicago Press,Chicago, IL, 1997.

[16] J. Schmeling, On the completeness of multifractal spectra, Ergod. Th. Dynam. Sys. 19 (1999), no. $6,1595-1616$

[17] A. Zygmund, Trigonometric series. Cambridge University Press, Cambridge,1959.

LAmFA, UmR 6140 CNRS, Université de Picardie, 33 rue Saint Leu, 80039 Amiens, FRANCE

E-mail address: ai-hua.fan@u-picardie.fr

lama, CNRS UmR 8050, Université Paris-Est, 61 Avenue du Général de Gaulle, 94010, Créteil Cedex, France

E-mail address: lingmin.liao@u-pec.fr

Department of Mathematics, Wuhan University, 430072 Wuhan, China

E-mail address: jhma@whu.edu.cn 